\title{
LOS MODELOS DE INTERACCIÓN ENTRE MÉDICO Y PACIENTE. DESCRIPCIÓN Y APLICACIÓN AL CONTEXTO DE LAS ENFERMEDADES POCO FRECUENTES*
}

\author{
ANTONIO M. BAÑÓN HERNÁNDEZ \\ Universidad de Almería \\ CySOC
}

\section{RESUMEN}

Este trabajo tiene como primer objetivo describir críticamente los distintos modelos de interacción entre médico y paciente. En las últimas décadas se ha hablado fundamentalmente del patrón biomédico, del prototipo psicosocial y del modelo combinado de ambos. El segundo objetivo será observar la aplicabilidad de estos modelos al contexto específico de las enfermedades poco frecuentes. Hablamos de enfermedades que afectan a menos de 5 por 10.000 habitantes y que tardan una media de 5 años en ser diagnosticadas. Apenas hay tratamientos disponibles para las aproximadamente 7.000 enfermedades de este tipo identificadas y el desconocimiento, incluso entre profesionales, sigue siendo notable. Este perfil repercute directamente sobre el marco en el que se desarrolla el intercambio de discursos entre profesionales y afectados. Además de la actualización bibliográfica correspondiente, hemos analizado las menciones que 9 personas adultas con enfermedades poco frecuentes han hecho de sus experiencias en este tipo de situaciones. Nos hemos detenido especialmente en los recuerdos que estas personas tienen en torno a la comunicación asimétrica con sus médicos de referencia.

PALABRAS CLAVE: Interacción médico-paciente, enfermedades poco frecuentes, análisis crítico del discurso.

\section{ABSTRACT}

The first objective of this work is to critically describe the different models of interaction between doctors and patients. In the last decades, the biomedical

\footnotetext{
* Este trabajo se enmarca dentro del proyecto de investigación "Lenguaje y cultura de la salud" (CS02014-61928-EXP), subvencionado por la Secretaría de Estado de Investigación, Desarrollo e Innovación del Ministerio de Economía y Competitividad. Está vinculado igualmente al contrato de investigación "Observatorio de las enfermedades raras. Banco de información”, subvencionado por FEDER y por el Ministerio de Sanidad, Servicios Sociales e Igualdad.
} 
pattern, the psychosocial prototype and the combined model of both have been discussed. The second objective will be to observe the applicability of these models to the specific context of rare diseases. We are referring to diseases that affect less than 5 per 10,000 inhabitants and take an average of 5 years to be diagnosed. There are hardly any treatments available for the approximately 7,000 diseases identified of this type, and ignorance, even among professionals, remains remarkable. This profile has direct repercussions on the framework in which the exchange of discourse between professionals and those affected is developed. In addition to the corresponding bibliographic update, we have analyzed the statements that 9 adults with rare diseases have made of their experiences in this type of situations. We have paid particular attention to the memories that these people have of their asymmetric communication with their reference doctors.

KEYWORDS: Doctor-patient interaction, rare diseases, critical discourse analysis.

\section{INTRODUCCIÓN Y OBJETIVOS}

En la década de los setenta del siglo pasado se inicia un debate público sobre la interacción de médico y paciente tanto en el terreno socio-político como en el de la investigación en el ámbito del discurso y de la comunicación (Papageorgiou, 2016: 22). Ese debate adquiere mayor intensidad en la década de los ochenta (Chenail y Morris, 1995: 2) y, naturalmente, llega a nuestros días. Es obligado mencionar la celebración de la Conferencia Internacional sobre Atención Primaria de Salud de Almá-Atá (6 al 12 de septiembre de 1978), en la que el concepto de salud quedó definido por la OMS como el estado de total bienestar físico, social y mental, y no simplemente como la ausencia de enfermedad (Cushing, 2016: 9), lo que, sin duda, obligaba a interrogarse por los objetivos de la consulta médica y añadía al debate actores anteriormente considerados complementarios como los asociados al mundo del trabajo, dado que el concepto 'trabajo decente' es básico en la consecución del mencionado estado de bienestar (Di Ruggiero et al., 2015). Además, movimientos como el feminista supusieron también un giro en cuanto a la comunicación de la salud en contextos en los que participaban las mujeres. Igualmente, los veteranos de guerra reclamaban una atención psicosocial más completa (Papageorgiou, 2016: 22). Pedro Laín ya había aludido en los años sesenta a la relevancia del componente psicosocial en la entrevista médica (1964: 75-78).

En la década de los setenta aparecieron libros tan emblemáticos como el de Labov y Fanshel (1977), así como los trabajos desarrollados desde la etnometodología médica y aplicados a interacciones orales (ten Have, 1995). De la misma manera, fueron publicadas investigaciones sobre la entrevista médica en revistas de prestigio en el ámbito del Análisis del discurso y de la Pragmática (por ejemplo, Conte, 1981). El uso de muestras 
de interacción grabadas (en audio o en vídeo) permitió ajustar mucho el tipo de investigación y mejorar las conclusiones (Cushing, 2016: 10). En realidad, las audiograbaciones para el estudio de la interacción clínica surgen ya en los años treinta en Estados Unidos, como recuerda Francesc Borrell (2004a: 301).

La consulta médica ha sido un género ampliamente estudiado y es claro que se trata de un tipo de interacción muy complejo y que acontece en entornos sanitarios muy diversos, como dice Alexia Papageorgiou (2016: 27). La bibliografía científica generada en el marco de los estudios del discurso y de la comunicación ha enfocado buena parte de sus esfuerzos en la descripción de los modelos de interacción, en los estilos y tonos, o en las dimensiones, fórmulas y estrategias (macroestructurales y microestructurales) observadas en las intervenciones de los profesionales sanitarios o de los pacientes y sus familiares. Se ha hablado, al menos, de tres modelos: el biomédico, el psicosocial y el combinado, al que se ha asignado la denominación 'biopsicosocial'. Vale decir, de cualquier forma, con Lorraine Noble, que es un error asumir que en la actualidad existe un acuerdo sobre una única y universal manera de relacionarse por parte de paciente y médico (2016: 57). Especialmente interesante resulta la observación de estos modelos cuando el intercambio discursivo se produce entre profesionales sanitarios y personas con enfermedades poco frecuentes.

El primer objetivo del presente trabajo es, pues, ofrecer una aproximación crítica al estado de la cuestión sobre esos prototipos comunicativos, así como sobre las repercusiones de los mismos en el discurso de sus protagonistas. El segundo objetivo será reflexionar sobre cómo las enfermedades poco frecuentes, dadas sus especiales características, se ajustan, en menor o mayor medida, a alguno de esos modelos. Para este segundo objetivo, hemos accedido también a la grabación de un grupo de discusión de nueve pacientes adultos con diferentes enfermedades poco frecuentes. En él se debatía sobre trayectoria personal y también en relación a las cuestiones que, en su opinión, eran mejorables. Inevitablemente, la interacción con sus médicos aparecía de forma explícita, en unos casos, e implícita, en otros. El grupo fue organizado por el Observatorio de las Enfermedades Raras de FEDER, cuya sede estaba establecida en ese momento en la Universidad Cardenal Herrera de Valencia ${ }^{1}$. Las enfermedades representadas en el grupo eran las siguientes: ataxia (T.1), síndrome de Behçet (T.2 y T.7), miastenia gravis (T.3), encefalomielitis miálgica (T.4), gastroparesia y pseudoobstrucción intestinal idiopática (T.5), déficit de 1 alfa antitripsi-

\footnotetext{
${ }^{1}$ Queremos mostrar nuestro agradecimiento al director del OBSER, Josep Solves, por la cesión de la grabación del grupo de discusión para este artículo y por su excelente gestión del Observatorio.
} 
na (T.6), esclerodermia (T.8) y osteogénesis imperfecta (T.9). La duración de la grabación fue de 2 horas, 20 minutos y 45 segundos. Fue transcrita al completo para la realización del presente artículo.

\section{LOS MODELOS DE INTERACCIÓN}

\subsection{Modelo biomédico o biofisiológico}

En el modelo biomédico o biofisiológico, se potencia la relación asimétrica entre el médico y el paciente o el familiar. Esa asimetría se establece no únicamente a partir de la evidente diferencia de conocimientos, sino también a partir de lo que podríamos denominar 'prestigio social'. Prestigio y asimetría confieren un mayor poder discursivo al profesional. El médico, en este marco de acción, puede no sentir el compromiso de ajustarse comunicativamente al paciente o hacer participar a este en las decisiones de diagnóstico o de terapia (Henzl, 1990: 78-79). El paciente ejerce como tal y la enfermedad es el centro del interés profesional; se concibe esta como una desviación de la norma conformada por variables biológicas que dejan huella en el cuerpo y que son objetivamente mensurables. Los médicos renuncian, de forma más o menos explícita, a interesarse por aspectos psicosociales y familiares del enfermo (Engel, 1977: 129-131). La enfermedad "ha sido separada de la persona y ha pasado a ser tratada como un ente propio" (Gadamer, 2001: 126). En 1977, George Engel afirmaba que este modelo biomédico era el dominante en el mundo occidental (pág. 130). Muchos médicos ya eran conscientes entonces de la existencia de excelentes modelos biomédicos que se mostraban especialmente débiles en cuestiones que para los pacientes eran fundamentales y que, habitualmente, tenían que ver con la comunicación (1977: 134). En 2002, van Dulmen afirmaba que en este prototipo médico y comunicativo se estaba abandonando cada vez más (pág. 243).

La hipertrofia del modelo conduce a la condescendencia (Street, 1991: 146) y al paternalismo (Charles, Gafni y Whelan, 1999: 652-653). Lo cierto es que el estilo discursivo normativo y más asimétrico se ha naturalizado en muchos casos (Gwyn, 2001: 69), porque se ha preferido la comodidad que, en cierto sentido, presupone (Silverman, 1987; Street, 1991: 148) o también por miedo a alterar el estatus de quien al fin y al cabo ha de dar su ayuda para ser curado (Silverman, 1987). Hay también muestras de lo que podríamos denominar 'interacción sobreasimétrica', como sucede cuando, por ejemplo, se suma a la formación técnica el dominio o no de una lengua (pensemos en personas inmigradas que acuden al médico) o los prejuicios de género (Gwyn, 2001: 75). 
Naturalmente, hay medios lingüísticos mediante los cuales se potencia el distanciamiento social entre médico y paciente (o familiares) (Henzl, 1990). La materialización discursiva de la asimetría en la interacción médico-paciente se ha descrito a partir de, por ejemplo, distintas fórmulas de control, tales como confirmar o no que de hecho se está enfermo y no se miente, o el poder de dar nombre a la enfermedad y facilitar el acceso a los medicamentos (Gwyn, 2001: 62). La libertad para auscultar físicamente también es un dato relevante (Heath, 1986 y 1989), como lo es la gestión del tiempo de la interacción (Thompson, 1990: 34). Desde otro punto de vista, la asimetría y el poder pueden observarse mediante la asunción casi absoluta por parte del médico de la iniciativa verbal y de las acciones que suceden en la consulta (Robinson, 2001), de la utilización de la risa (Robinson, 2001) y de la sonrisa (Haakana, 2001 y 2002) o a través del uso preferente por parte del profesional de las preguntas (especialmente cerradas) (Henzl, 1990: 89; Frankel, 1990). Lo mismo valdría decir de la ausencia de información al paciente con respecto al proceso de razonamiento o de deliberación seguido para llegar a un diagnóstico o a un tratamiento (Charles, Gafni y Whelan, 1999: 656), o de la tendencia a obviar las informaciones de las circunstancias sociales o personales a las que hacen referencia los pacientes (Treichler et al., 1984: 73; von RafflerEngel, 1989: 5; ten Have, 1991: 140; Clark y Mishler, 1992; Beach, 1995: 256257 y Jones, 2001). La asimetría hace que, con frecuencia, no se atienda a cuestiones esenciales como, por ejemplo, los sentidos asignados a la palabra 'dolor' o a la palabra 'fatiga' por parte de personas enfermas de Parkinson o de fatiga crónica (Olson et al., 2015). Además, la noción de esperanza es entendida, en muchos contextos, de forma muy diferente por parte de pacientes o de familiares y de profesionales sanitarios, que ven en el uso de esa noción más bien una muestra de estar 'fuera de la realidad' (Roscigno et al., 2012). En el marco de la 'construcción médica de la esperanza', cada persona posee una idea de la misma que puede ser creada, modificada o destruida (Eliott y Olver, 2002: 174). Igualmente, por su relevancia, hemos de mencionar las distintas significaciones que, para médicos, investigadores y pacientes, podemos asignarle al concepto de riesgo (Opsal et al., 2016).

También es muestra de poder social y discursivo el silencio del médico o la interrupción del turno del paciente (Frankel y Beckman, 1984; Stratford, 1998; Koch-Weser et al., 2009: 380) o la capacidad para cambiar de tema (Gwyn, 2001: 67), así como el uso de jerga médica incomprensible para el paciente o para el familiar (Koch-Weser et al., 2009) quienes, con frecuencia, no reaccionan pidiendo aclaraciones (Thompson, 1990: 30-31).

En realidad, lo que hacen los facultativos es, en algunos casos, aplicar, sin apenas interrogarse sobre sus bondades y sobre sus defectos, el método 
de trabajo que, durante generaciones, han aprendido en las facultades de medicina y que supuestamente aseguraba una mayor objetividad y cientificidad (Cassell, 1989: 14-15). Ha de llamar la atención el hecho de que los estudiantes de medicina, conforme avanzan en sus estudios, aprenden a ignorar cada vez más el discurso de los pacientes y a convertir las conversaciones con ellos en interrogatorios (Weston y Lipkin, 1989: 56).

Puede suceder que este modelo sea aceptado por todos los actores implicados, pero también puede suceder que el estilo de interacción no sea colaborativo, por distante, por poco informativo o por desvinculado de la realidad social y emocional del paciente. $\mathrm{O}$, desde otro punto de vista, por considerar que la intensificación del modelo centrado en el paciente puede generar solapamientos con tareas propias de los profesionales, como han analizado Lisa Mikesell y Elizabeth Bromley (2012). El conflicto y las relaciones de poder y solidaridad es foco de interés para los sociolingüistas (Henzl, 1990: 80; Elwyn et al., 1999); un conflicto que puede iniciarse incluso antes de la consulta, en el tiempo de espera ${ }^{2}$. El desacuerdo puede hacerse explícito o proponerse de forma más bien implícita. A mayor grado de desacuerdo en la gestión comunicativa, menor será la adherencia a los consejos médicos o a los tratamientos indicados. Quienes no aceptan el diagnóstico o quienes tienen enfermedades crónicas propenden más a la no adherencia al tratamiento indicado (Britten et al., 2004). Si trasciende esa insatisfacción puede llegar a quejas informales, a quejas formales, a peticiones de segunda opinión o, en los casos más graves, a violencia física o verbal ${ }^{3}$. La existencia de errores médicos puede detonar alguna de estas reacciones.

El debate sobre la existencia de una desafección creciente hacia los profesionales médicos está abierto, desde hace años, sin duda (von RafflerEngel, 1989: 3), pero, como en casi todo, la responsabilidad del éxito comunicativo no puede recaer únicamente en el profesional. Suele aludirse a los 'enfermos complicados' como aquellos que tienen un perfil pluripatológico, "que no quieren nada" o que no son capaces de transmitir bien lo que les sucede 4 . También se habla con frecuencia de "pacientes difíciles', que se estima que pueden ser entre un 1 y un 3 por ciento del total asistido por los médicos en una consulta. Como suelen ser "hiperfrecuentadores", al menos un $10 \%$ de las consultas pueden definirse como difí-

\footnotetext{
${ }^{2}$ Rosalía Sierra, "La insatisfacción de los pacientes se suele generar fuera de la consulta”, Diario Médico, 6-12 de junio de 2016, pág. 8 .

${ }^{3}$ J. A. G, J. P. P. y A. N., "El marido de una paciente propina una paliza a un médico de Cartagena", La Verdad, 10 de diciembre de 2011, pág. 24. 2003.

4 Joaquín Mayordomo, "El desafío de los enfermos complicados", El País, 15 de abril de
} 
ciles (Ágreda y Yanguas, 2001: 66). Son pacientes, por ejemplo, que no quieren hablar de asuntos importantes para su diagnóstico o tratamiento, que no confían en el médico, que no aceptan el diagnóstico o que no quieren ser dados de alta (Borrell, 2004a: 198-208). Ahora bien, al profundizar en los tipos de contextos en los que, según el profesional, se está ante un paciente de este tipo, se puede llegar a colegir que los factores que conducen a etiquetar a una persona así pueden estar en el propio enfermo, pero también puede derivarse de los sentimientos o emociones que los pacientes generan en el profesional (Ágreda y Yanguas, 2001: 66). De hecho, los médicos que "tienen menos capacitación en técnicas de comunicación y abordaje psicosocial refieren un mayor número de pacientes difíciles" (Bellón, 2001: 8).

Wayne Weston y Mack Lipkin (1989: 50) ofrecen una interesantísima distinción entre el deseo de curar y el deseo de cuidar como dos objetivos sobre los que los médicos deberían reflexionar, para intentar equilibrarlos. Parece que, por lo general, los profesionales de la medicina han delegado la segunda responsabilidad en otros actores que participan en las interacciones en contextos sanitarios. José F. Prieto lo ha expresado también aludiendo a los profesionales de nuestro ámbito que apenas reflexionan sobre su obligatoria tarea de persuasión, y, cuando lo hacen, asignan connotaciones negativas al término persuasión, como si persuadir fuese propio sólo de curanderos y charlatanes (2001: 126).

\subsection{Modelo psicosocial}

Estudiosos de la comunicación en contextos sanitarios, como Hydén y Mishler (1999: 185), hablaban de un cambio radical en el discurso médico dominante, basado, por ejemplo, en el aumento de información disponible, también para el enfermo y para sus familiares. En cualquier caso, este cambio de modelo ha pasado, claro está, por distintas fases. Inicialmente, se hablaba de "modelo clínico transformado" (McWhinney, 1989: 37-38). Se ha hablado también de "modelo relacional" (Kushner, 1981). Lo que es claro para muchos es que la dimensión moral y también la simbólica que acompañan al hecho mismo de estar enfermo son desafíos a los que el médico ha de intentar dar respuesta no sólo como técnico, sino también como persona (Mordacci y Sobel, 2004: 106). Se busca una relación que propenda a la simetría comunicativa, sin que eso signifique necesariamente igualdad desde el punto de vista de los conocimientos técnicos o científicos, ya que, por lo normal, no se comparten, para empezar, registros con el mismo repertorio lingüístico (Henzl, 1990: 81). La equidad comunicativa significa, al menos, que el paciente puede expresar libremente sus preocupaciones, 
preguntar, proporcionar información que considera relevante más allá de la mera respuesta a las preguntas recibidas (Stivers y Heritage, 2001) e incluso ofrecer opiniones sobre tratamientos (Street, 1991: 148). El punto de partida habrá de ser el reconocimiento de que los pacientes llegan al encuentro con su médico con sus valores, creencias, miedos y experiencias sobre la enfermedad; e interpretarán la información proporcionada por el profesional a partir de esas variables (Charles, Gafni y Whelan, 1999: 655). El profesional médico ha de saber que también ha de cumplir la función de 'prescriptor de información terapéutica'; es decir, información que mejore la salud física y mental de los pacientes. Esa función incluye la orientación y el acceso a datos y propuestas relevantes (Chesser et al., 2012). La entrevista motivacional está relacionada justamente con esa atención centrada en el paciente (Lizarraga y Ayarra, 2001).

En este tipo de interacciones, hay muchas presuposiciones compartidas, pero también hay presuposiciones no compartidas (von Raffler-Engel, 1989: 1). De lo que se trata es de compartir responsabilidad en el proceso de diagnóstico, en la asunción de la enfermedad y también en la aceptación y cumplimiento de las medidas terapéuticas asignadas. Es lo que se conoce como decisión compartida y elección informada (Gwyn, 2001: 79). En relación al tratamiento, la decisión compartida es de aplicación aún más difícil (Charles, Gafni y Whelan, 1999: 651). Algunas de las características del modelo centrado en el paciente serían las siguientes:

a) el doctor sugiere un plan de ayuda al enfermo;

b) se muestra abierto de mente;

c) se interesa en el enfermo como persona;

d) ofrece al enfermo la oportunidad de decir lo que piensa;

e) presta atención a lo que opina el paciente;

f) es claro y utiliza un lenguaje sencillo (Swenson et al., 2004: 1074).

No está de más recordar que la interacción centrada en el paciente o el empoderamiento del paciente es más que hacer visible a ese paciente ofreciéndole la posibilidad de mostrar su discurso (Malson et al., 2004: 478). En este sentido, el consentimiento informado ha sido mencionado en algunos casos como un avance esencial en la autonomía del paciente y en la transición de modelos paternalistas a otros más participativos (Béry, 2005). Ahora bien, los problemas de comprensión del consentimiento informado también son un hecho cierto (Krosin et al., 2006). En general, es posible que el nivel de comprensión de los pacientes sea sobrevalorado en muchas ocasiones; y de hecho, suelen confiar en lo que se les dice e incluso, a veces, delegar la decisión en otros (Hall et al., 2012: 534).

En todo caso, puede que, al final, se trate más bien de una decisión compartida pero orientada hacia la preferencia mostrada explícita o implícita- 
mente por el médico (Gwyn, 2001: 79); téngase en cuenta que a la hora de ofrecer información sobre alternativas terapéuticas, la información actualizada del profesional es determinante y la decisión compartida se tomará a partir de esa información (Charles et al., 1999: 654). Por otra parte, hay quien se pregunta si en este marco se ha de incluir también el hecho de compartir la incertidumbre, ya que eso puede generar desconfianza y nerviosismo en los pacientes. Éstos se muestran muy atentos a la aparición, durante su interacción con los médicos, de frases prototípicas como las siguientes: 'I'm not sure about this', 'I haven't come across this before', 'I don't know', 'I think it might be' (Dobson, 2002: 1319).

Las enfermedades crónicas generan un espacio propio para la construcción discursiva colaborativa de los cuidados reclamados por los pacientes (Engeström et al., 2003). El factor tiempo es esencial en el éxito de la decisión compartida (Charles, Gafni y Whelan, 1999: 655); y el prototipo de interacción de 6 o 7 minutos de media consignado a la atención de cada paciente no favorece tal éxito (Gwyn, 2001: 79; Bellón, 2001: 11). El tiempo, como es bien sabido, es un factor importante en la manifestación de quejas por parte de los usuarios (von Raffler-Engel, 1989: 5; Borrell et al., 2011: 218-220). Extender el tiempo de la consulta ha sido una petición constante de los profesionales y de los pacientes, aunque todo dependerá del tipo de patología y de otros factores contextuales (Lee, 2002: 1242).

El paciente ejerce, pues, en este modelo, más de agente y el núcleo del interés pasa de la enfermedad a la persona enferma (Sharf y Street, 1997; Bennett e Irwin, 1997). Hay quien ha vinculado este modelo a una redefinición de los roles del médico y del paciente en términos de proveedor de servicios de salud y cliente o consumidor (Sharf y Street, 1997: 4). El cuidado psicoterapéutico ya ha avanzado mucho en este modelo (Levenstein $e t$ al., 1989: 110). Y también el trabajo social; su influencia y la aparición de movimientos en los que se solicitaba una atención centrada en la familia en contextos pediátricos, ya en los años cincuenta del siglo pasado en Estados Unidos, son precedentes de este nuevo modelo (Korsch, 1989: 246-247). En este sentido, también existe quien se pregunta si estamos conduciéndonos hacia un modelo consumista en el que las relaciones de poder simplemente se están revirtiendo y el médico acaba adoptando un rol más pasivo, accediendo, sin más, a las peticiones de los pacientes (sobre tratamientos, segundas opiniones, derivaciones a hospital, etc.) (Cushing, 2016: 16).

El modelo psicosocial reclama unas habilidades comunicativas de las que los profesionales no siempre disponen y de cuya importancia no siempre se es consciente (Borrell y Epstein, 2004); es entonces cuando hay que pensar en una formación específica de este tipo, que implica saber comunicar y hacerse entender (von Raffler-Engel, 1989: 14), así como manejar convenientemente los aspectos emocionales y psicosociales de la comunica- 
ción y de la interacción médica (van Dulmen, 2002: 248). Peter Maguire y Carolyn Piceathly (2002: 697) consideran que esta preparación específica tendría cinco ventajas:

1. Los doctores son capaces de identificar los problemas de los pacientes de forma más precisa.

2. Los pacientes están más satisfechos porque pueden comprender mejor sus problemas, las investigaciones en curso y las opciones de tratamiento existentes.

3. Mejora la adherencia de los afectados al tratamiento.

4. Se reducen los niveles de ansiedad y vulnerabilidad en los pacientes.

5. Mejora igualmente el bienestar de los propios profesionales sanitarios.

En lo que respecta a la comunicación paraverbal, algunos especialistas han mencionado, por ejemplo, el valor del contacto visual, del tono y de la entonación en estas interacciones tendentes a una mayor simetría entre médico y paciente (Zoppi y Epstein, 2002; Blanck et al., 1986).

Las habilidades comunicativas son importantes en la transmisión de malas noticias (Gómez, 2000), pero, paradójicamente, numerosos conflictos ocurren al dar 'buenas noticias' (Maynard y Heritage, 2005: 432). La formación en elementos microestructurales como el uso de atenuadores discursivos es muy útil en la gestión de la interacción del médico con sus pacientes (Maynard y Heritage, 2005: 432). Más específicamente, por ejemplo, los doctores han de aprender a promocionar las preguntas iniciadas por los pacientes, especialmente en áreas tan sensibles como la oncología (Murtagh et al., 2013).

Esta formación es cada vez más solicitada por los propios médicos y los estudiantes de medicina, si bien aún sigue estando vigente entre profesionales y estudiantes la siguiente pregunta: ¿Debo priorizar la resolución del problema médico o realizar una escucha atenta y activa? (Aper et al., 2015). En cualquier caso, los pacientes también han de formarse (Aibar, 2009: 184) y mejorar, por ejemplo, su conocimiento de la terminología médica (Thompson y Pledger, 1993). Y algunos están muy formados, de hecho. Por eso se habla de pacientes expertos o empoderados (Zoppi y Epstein, 2002: 323), lo que corresponde a pacientes muy bien informados que dan nombre a programas universitarios ${ }^{5}$ y que han sido calificados como "pacientes activados' (o 'activos') o como 'motores de cambio en la Sanidad' ${ }^{6}$. Tam-

\footnotetext{
${ }^{5}$ Redacción, "El programa del 'paciente experto' llega a la universidad", La Verdad, 9 de diciembre de 2012, pág. 10.

${ }^{6}$ Carmen Torrente, "Pacientes, motor de cambio en la Sanidad", 23-29 de mayo de 2016, pág. 54.
} 
bién sobre esto hay una doble mirada por parte de profesionales; la de quienes creen que es un escenario ideal y la de quienes creen que es una pesadilla. No es casualidad que una editorial del prestigioso British Medical Journal (BMJ) se titulase justamente así: “'Expert patient' - dream or nightmare?" ". En efecto, habrá quien vea en esta figura una muestra de paciente disruptivo o difícil (Schmidt et al., 2017). Y habrá quien crea que, gracias a ella, la relación médico-paciente mejorará ya que tratará con personas interesadas y con ganas de gestionar su propia vida, especialmente si hablamos de enfermedades crónicas ${ }^{8}$. Planteado como un reto al que se enfrentan los profesionales sanitarios, puede que el proceso de adecuación comunicativa entre médico y paciente presuponga, de entrada, una cierta incomodidad ${ }^{9}$. Como el término 'experto', en cualquier caso, ha suscitado recelos, otras alternativas léxicas se han propuesto, como recuerda la editorial de BMJ: paciente autónomo o paciente implicado, entre otras (2004: 724).

La 'autonomía del paciente' tampoco es un concepto carente de polémica (Nessa y Malterud, 1998). Esta clase de pacientes encaja bien en la tendencia al 'empoderamiento' de los colectivos sociales, incluidos los relacionados con la salud y la enfermedad (Sharf y Street, 1997: 6; Bennett e Irwin, 1997: 90). Es evidente que Internet ha supuesto una herramienta decisiva en la conformación de este tipo de pacientes (Cushing, 2016: 15). Christopher Candlin (2000: 1) habla de la reinvención del paciente y de su repercusión en la comunicación en contextos sanitarios. Una reinvención motivada, según él, por las siguientes circunstancias:

a) una intensificación general de la representación de los discursos individuales;

b) unas mayores inversiones públicas en salud;

c) la necesidad de los profesionales sociosanitarios de conocer mejor a sus pacientes, especialmente si están en una situación grave y crónica; y

d) el acceso cada vez mayor a fuentes de información fiables (en Internet y también en medios de comunicación convencionales).

Una hipertrofia del modelo psicosocial lleva a la hiperresponsabilización del enfermo (o de sus familiares) en la gestión de la enfermedad (o en la búsqueda de alternativas terapéuticas) o a la derivación de problemas físicos al ámbito exclusivamente psicológico (Borrell, 2004a: 88); y por de-

7 Vol. 328, 2004, págs. 723-724.

8 Joan C. March, “¿Son necesarias nuevas formas de relación profesional-paciente?”, Diario Médico, 23-29 de mayo de 2016, pág. 58.

${ }_{9}$ Isabel Perancho, "Médicos y pacientes deberán aprender a relacionarse de forma igualitaria”, El Mundo-Salud, pág. 8, 21 de junio de 2003. 
fecto, de nuevo (como en el modelo biomédico) a la comodidad, ya que se asumen menos responsabilidades. Este modelo tampoco asegura que la interacción sea colaborativa y, de hecho, puede generar conflictos, dado que hay pacientes que, como decíamos, prefieren no tener un rol activo ni asumir responsabilidades (Gwyn, 2001: 79; Swenson et al., 2004; Hsieh et al., 2016), o que incluso llegan a dudar de la preparación de los médicos, dado que la decisión compartida es percibida como prueba de incertidumbre y debilidad antes que como indicador de colaboración (Gwyn, 2001: 80). Pero el conflicto puede llegar también si la simetría se entiende como la eliminación de protocolos en el trato que sorprenden a alguno de los interlocutores; pensemos, por ejemplo, en el tuteo dado a las personas mayores hospitalizadas que, a veces, no entienden ese trato o, en general, el uso de los apelativos (Morant, 2016). Las fórmulas de cortesía o descortesía y las distintas interpretaciones que de esos dos conceptos hacemos las personas al hablar cumplen una función evidente en este sentido (Amer y Fischer, 2009).

\subsection{Modelo biopsicosocial y reticular}

Lo cierto es que es posible mantener, al mismo tiempo, entre los médicos y sus pacientes una relación simétrica y asimétrica. Marisa Cordella (2000), a partir de un corpus de entrevistas semidirigidas desarrolladas en un centro médico chileno, estudió la materialización de esta doble relación en aspectos comunicativos concretos: la muestra de respeto hacia el paciente, el uso de saludos tendentes paulatinamente hacia la informalidad, la elusión de conflictos comunicativos, la sensata incorporación de las opiniones e incluso de las propias palabras del paciente, la escucha alejada de impaciencias, el rechazo de la jerga demasiado específica y su sustitución, siempre que sea posible y conveniente, por coloquialismos, serán algunas de las fórmulas generadoras de aproximación. Por otro lado, la interpretación de las pruebas o las explicaciones complementarias a partir de las dudas mostradas por el paciente serán algunas de las muestras de poder.

Parece claro, por lo tanto, que un modelo biopsicosocial, que permita acomodarse a las circunstancias comunicativas específicas y al contexto de interacción, puede ser la opción más equilibrada, en un período de transición o de solapamiento de modelos como el vivido en las últimas décadas (von Raffler-Engel, 1989: 1). En realidad, el modelo biomédico también habría de tener en cuenta que cada interacción es distinta y se negocia de forma diferente por parte de médicos y pacientes según se esté en una fase u otra de la consulta, lo que relativiza el concepto mismo de asimetría (ten Have, 1991).

La 'acomodación' en la comunicación entre médico y paciente es un concepto de largo uso a partir de su aparición como proceso clave para 
los estudios sociolingüísticos (Coupland et al., 1988). La acomodación tiene éxito si se contextualiza adecuadamente la comunicación a partir de, al menos, los siguientes elementos: ¿en qué clase de sistema sanitario se produce la interacción (público o privado)? (Ainsworth-Vaughn, 1998), ¿qué tipo de enfermedad?, ¿cuántos participantes?, ¿qué tipo de participantes (informados o no; niños, adolescentes, adultos o personas mayores)?, ¿en qué fase se está?, ¿ha habido otras interacciones previas?, ¿cuál es el 'historial comunicativo' del paciente y del profesional?, ¿dónde se desarrolla la interacción (hospital y estando hospitalizado, en urgencias, en la consulta, etc.)?, ¿cuánto tiempo durará previsiblemente la interacción? Richard L. Street ya se ocupó de la acomodación en consultas médicas en un trabajo incluido en un libro clásico en el estudio de este concepto: Contexts of Accommodation. Developments in Applied Sociolinguistics (1991). Entre las variables estudiadas, incluía la edad del paciente (que llevaba en ocasiones, por cierto, a una sobreacomodación basada en la mirada estereotípica a partir de la cual se infravaloran las habilidades lingüísticas y cognitivas de los pacientes), la ansiedad del enfermo (algo que varía enormemente de una persona a otra, que tiene que ver con la gestión de la incertidumbre, y sobre la cual la comunicación no verbal del médico tiene mucha incidencia, para bien y para mal), el historial de relaciones comunicativas entre ese médico y ese paciente (puede ocurrir que sea un paciente habitual o que sea un paciente que acude por primera vez), la educación y la clase social del afectado (los doctores dedican más tiempo y proporcionan más información a los pacientes con formación universitaria o de clase económica alta, además de mostrarse, por lo normal, más empáticos con ellos, entre otras cosas porque comparten, también en buena medida, códigos culturales y comunicativos), y la identidad sexual del paciente (el médico dedica más tiempo y da más información a las pacientes mujeres que a los hombres; o tiene que realizar más negociaciones a partir de patrones de carácter cultural, Fisher y Groce, 1985). Todos los factores mencionados son relevantes, aunque tal vez la $e d a d$ sobresale de forma especial. La interacción comunicativa de los profesionales de la medicina con adolescentes, por ejemplo, ha sido vista como un área específica que reclama una atención propia (Cornellá y Llusent, 2002; Beresford y Sloper, 2003). Pero algo parecido podemos decir de la interacción con pacientes ancianos, por sus dificultades para obtener y aprehender la información que se les proporciona, y por sus limitaciones a la hora de reaccionar adecuadamente ante los mensajes incomprendidos (Cegala et al., 2001: 1505; Coupland et al., 1994). Especialmente, en urgencias médicas (Majerovitz et al., 1997).

Borrell ofrece una mirada equilibrada, en nuestra opinión, en relación a los modelos comunicativos e insiste en la importancia de atender a la práctica concreta, a la 'entrevista clínica viva', que difícilmente atiende a 
marcos interpretativos estrechos (2004a). De hecho, propone hablar más que de modelos esencialistas, de "perfiles profesionales", lo que significa "renunciar a grandes modelos explicativos de casi todo, pero tiene la ventaja de poner bases sólidas a los pequeños avances que se puedan producir" (2004b: 30). Ciertamente, se trataría de asumir que "las posiciones pragmáticas que apuntaban por rescatar de cada escuela lo que de verdad era aplicable con ventaja en las consultas del clínico han acabado por imponerse" (2004a: ix). En cualquier caso, propone, por su parte, un modelo emotivo-racional que sea cuidadoso con las dos fases esenciales de la interacción clínica (exploratoria y resolutiva, 2004a: 17) y que preste una atención especial al contexto, lo que supone, entre otras cosas, la estimulación de la comunicación, la identificación de intencionalidades y, muy especialmente, la capacidad para encuadrar y reencuadrar la entrevista según vaya avanzando la misma (2004a: 18). Rechaza así la batalla de ideologías (2004b: 30) que analiza todo en términos de 'buenos' y 'malos' a partir de conceptos como 'centrado en el paciente', 'abordaje integral', 'abordaje negociador', etc. (2004b: 30). Tal vez, el término "alianza terapéutica” (Borrell, 2004a: 175) expresa, mejor que ningún otro, el proceso de acomodación discursiva y emocional entre médicos y pacientes.

Por nuestra parte, proponemos hablar también de un componente reticular añadido al biopsicosocial. Es decir, la interacción entre los profesionales sanitarios y los pacientes o sus familiares ha de desarrollarse en el marco de otras interacciones ya desarrolladas con otros profesionales que hayan atendido a esa persona. De esas interacciones, hay que ser consciente que han de estar coordinadas entre sí. Esto es especialmente importante cuando hablamos de enfermedades complejas como son, en su mayor parte, las enfermedades poco frecuentes.

\section{LA INTERACCIÓN DE LOS PROFESIONALES SANITARIOS CON LAS PERSONAS CON ENFERMEDADES POCO FRECUENTES Y SUS FAMILIARES}

\subsection{Reflexiones generales}

El retraso diagnóstico es una de las características fundamentales de muchas enfermedades poco frecuentes. En el Estudio de necesidades sociosanitarias de las personas con enfermedades raras en España (ENSERIO), publicado en 2009 por la Federación Española de Enfermedades Raras (FEDER), de hecho, se indicaba que el 23,52\% tardaba en llegar al diagnóstico definitivo entre 1 y 3 años; un 17,75\% tardaba entre 4 y 9 años; y el 21,01\% había de esperar diez años o más. El resto tenía un tiempo de espera inferior a un año (2009: 44). La muestra total ascendía a 715 personas. Este mismo 
año 2017 va a ser presentado un nuevo informe ENSERIO en el que las cifras indican que un $89,15 \%$ dispone de un diagnóstico confirmado, el $7,61 \%$ tiene un diagnóstico no confirmado y el 3,24\% no dispone de diagnóstico. El tiempo transcurrido para llegar al diagnóstico definitivo fue de entre 1 y 3 años para el 18,91\%; de entre 4 y 9 años para el 18,08\%; y de 10 años en adelante para el 18,65\%. El resto de personas aguardó menos de un año (FEDER, 2017: en prensa). Ha descendido, pues, el número de personas que reciben el diagnóstico después de un año de iniciadas las pruebas, aunque siguen siendo más del $50 \%$. Ha subido, con todo, el porcentaje de pacientes que han de esperar entre 4 y 9 años.

Sobre las causas del retraso diagnóstico, la referida mayoritariamente es la falta de conocimiento de la enfermedad por parte de los profesionales $(72,52 \%)$ a la que se suman otros menos relevantes como las gestiones administrativas o la evaluación económica del hospital o de las Comunidades Autónomas, por ejemplo. En relación a las consecuencias, el 31,26\% alude al agravamiento de la enfermedad o de los síntomas, el 29,37\% a no haber recibido tratamiento, el $17,90 \%$ a haberlo recibido inadecuadamente y el $15,30 \%$ a la necesidad de recibir atención psicológica. De nuevo, puede presuponerse la trascendencia de todas estas cuestiones en el discurso oral de pacientes y familiares, un 30\% de los cuales, además, afirma haberse sentido discriminado en la vida cotidiana y en las relaciones sociales.

Todas estas cifras tienen una repercusión directa sobre la interacción que los profesionales desarrollan con los pacientes o con sus familiares. Los trabajos sobre aspectos socio-comunicativos en torno a las enfermedades raras son muy escasos en el panorama internacional (Requena y Bañón, 2016; Requena, Arcos y Bañón, 2014; Bañón y Fornieles, 2011; Bañón, 2007). Pero ya empiezan a aparecer algunas investigaciones en torno a la repercusión que el perfil específico de este tipo de patologías puede tener en la interacción médico-paciente (Budych et al., 2012). La escasez de conocimientos incluso por parte de los profesionales o el especial perfil psicológico asociado a una interacción en la que se comunica un diagnóstico de una enfermedad sin cura son dos elementos determinantes, como también lo es el que los pacientes, aunque en general puedan preferir que sean sus médicos los que lleven la iniciativa, se vean obligados en muchas ocasiones a convertirse en casi especialistas de la enfermedad y a estar actualizados en torno a las posibles terapias en fases iniciales, a pesar de que carezcan de una formación específica en aspectos médicos y científicos (2012: 155). La adaptación a un modelo de interacción más simétrico puede deberse, pues, a las características propias de las enfermedades poco frecuentes, siempre que el enfermo o sus familiares tengan la competencia para asumir esa responsabilidad. 
Por eso, hay momentos en los que se produce una cierta inversión de roles; es decir, los afectados se convierten en pacientes expertos, tras haber adquirido información especializada sobre su patología que, por lo normal, intentaban compartir con sus doctores de referencia (2012: 159). Esta situación puede generar conflictos, dado que los profesionales suelen ser bastante reacios a esta inversión de roles (2012: 156). Las personas entrevistadas por Budych et al. (2012: 157) aludían, por ejemplo, a médicos que mostraban una actitud arrogante hacia el paciente y que, en ocasiones, interpretaban el 'empoderamiento' de los pacientes como una amenaza a su 'poder social' (2012: 159). En todo caso, a veces sí se relataba la adaptación y modificación de los roles durante el proceso de interacción.

Entre los testimonios de los pacientes con enfermedades raras, encuentran patrones de interacción paternalista (el médico decide, sin apenas contar con la opinión del paciente, y éste, en muchas ocasiones, asume esa situación), patrones de interacción colaborativa (en los que el médico reconoce el nivel de conocimiento de los pacientes y éstos, a su vez, reconocen también el rol profesional del médico), el patrón de interacción dirigida por el paciente (es éste el que dirige al médico, que no tiene conocimiento de la enfermedad a la que se enfrenta) y, finalmente, el patrón de interacción basada en la confrontación (el paciente evalúa e incluso llega a desafiar la competencia del médico, y éste reacciona aumentando su nivel de decisión e ignorando las sugerencias y las preferencias del paciente). Las características personales del médico o de los pacientes pueden influir en el tipo de patrón utilizado. Es evidente que las habilidades comunicativas del médico tendrán un protagonismo especial en este contexto, como lo tendrá el grado de expectativa que tiene el paciente con respecto a su doctor o la empatía que pueda mostrar ante situaciones de especial gravedad (2012: 159-160). Por los datos disponibles, los pacientes que sufren una enfermedad poco frecuente, degenerativa y sin cura posible muestran por lo normal una predisposición mayor a interactuar colaborativamente con sus médicos, y también toman la iniciativa en la búsqueda de información relevante para su enfermedad (2012: 161). Esto no asegura estar preparado necesariamente para recibir el diagnóstico. Un estudio que se realizó con padres de afectados por el síndrome de Turner desveló que aunque a veces los padres se informan antes de recibir el diagnóstico, en el momento de la comunicación del mismo se presentan como 'no preparados' para recibir la noticia (Starke et al., 2002).

Algo también habitual en la interacción entre actores con un perfil social y sanitario tan especial es, como decíamos, el enfrentamiento. Uno de los motivos, en origen, puede ser la dificultad para conseguir el diagnóstico y, por lo tanto, la presencia de síntomas que no consiguen ser explicados desde el punto de vista médico de forma convincente. Sarah Peters et al. (1998: 559-560) han estudiado este perfil de pacientes que suelen 
tener un contacto muy frecuente con profesionales médicos de especialidades variadas y que acaban adquiriendo un aceptable dominio de la terminología y de la manera de pensar de los profesionales. El relato de los pacientes entrevistados para la realización de la investigación coincide en ocasiones con el de los pacientes con enfermedades poco frecuentes y, así, se alude, por ejemplo, a profesionales con pocos conocimientos. Los médicos también muestran explícita o implícitamente valoraciones negativas de los pacientes, a los que atribuyen sus problemas físicos a ansiedad o a depresión (1998: 562). Estos pacientes se quejan también del hecho de que no se haya conseguido dar un nombre a lo que les pasa (1998: 563), igual que suelen manifestar quienes tienen enfermedades minoritarias. De hecho, son muchas las personas sin diagnóstico.

Otro motivo de este enfrentamiento es el derivado de la no comunicación de posibles tratamientos que estén en desarrollo para la enfermedad debido al desconocimiento del propio profesional sanitario, algo que puede llevar incluso hasta recomendar un tratamiento incorrecto para la enfermedad en cuestión (Rivera-Navarro, 2009: 18; Halpin, 2011: 862).

La tensión emocional es algo casi inevitable en la comunicación del diagnóstico. Esa tensión es mayor aún si las noticias que se han de comunicar no son buenas (Fallowfield y Jenkins, 2004; Schaepe, 2011). O si el paciente y su familia han tenido que esperar mucho tiempo hasta llegar a ese diagnóstico. Este tipo de dolencias poco frecuentes son causa de muerte en el $35 \%$ de los niños menores de 1 año, del $10 \%$ entre 1 y 5 años y del $12 \%$ entre los 5 y 15 años de edad (Servicio de Epidemiología de la Comunidad de Madrid, 2008: 7).

Como el pronóstico suele acompañar al diagnóstico, hemos de pensar en situaciones en las que, a la tensión derivada del hecho de saber que se padece una enfermedad poco frecuente y que esa etiqueta puede conllevar una cierta estigmatización social, hay que añadir el saber que no hay tratamientos disponibles, que son enfermedades que acompañarán toda la vida y que, a veces, tienen un carácter degenerativo. Ciertamente, esta tensión no afecta sólo a los pacientes y familiares. También se refleja en los médicos, quienes pueden disponer de poca información sobre la patología, sobre posibles iniciativas de investigación o sobre asociaciones de pacientes que puedan ayudar a estas personas recién diagnosticadas (Firth, 1983: 701).

\subsection{Menciones a la interacción entre médico y pacientes en el discurso de personas con una enfermedad poco frecuente}

Ya hemos visto que el diagnóstico es un elemento sobre el que gravita buena parte del discurso de las personas afectadas ya que tiene, claro está, 
una gran intensidad semiocomunicativa (Laín, 1982; Chiong, 2004). La expresión temporal y espacial permite representar bien el progreso o la ralentización en la búsqueda de diagnóstico o de tratamiento (continuidad), por un lado, o el retroceso y la detención en esa misma búsqueda (discontinuidad), por otro.

Aunque también hay referencias a interacciones más simétricas, nos vamos a detener en este apartado en la denuncia por parte de los testigos de comportamientos discursivos propios del modelo biomédico, más asimétrico.

Así, por ejemplo, se alude a la ausencia de explicaciones y a la falta de aceptación por parte de los profesionales del desconocimiento. El uso del verbo 'pasar' o de la expresión 'buscarse la vida' es muy significativo en estos dos ejemplos:

(1) Antes pues los médicos pasaban; te decían que estabas normal. No sabían ni que existía, pero te decía que era normal (T.1).

(2) Ellos me diagnostican y me dicen 'No hay tratamiento. Es crónico. Es irreversible. Es invalidante'; esas palabritas mágicas que te dicen, y bu-, a buscarse la vida” (T4).

De igual forma, la infravaloración de los síntomas sentidos por los pacientes forma parte de este mismo modelo, expresado en ocasiones mediante fórmulas cercanas al paternalismo. El uso de imperativos en el discurso recordado, la presencia de la palabra 'excusa' frente a, pongamos por caso, 'explicación' o ‘interpretación', o la aparición a destiempo del halago junto con el valor conclusivo de 'Hala' refuerzan discursivamente esta estrategia:

(3) Me dan el diagnóstico y me dicen 'Hala, vete a casa y: y sé positiva'. Esa fue la frase, con lo cual dices: ‘¿Umm?'. Que yo a ese hombre en el momento que pueda lo voy a demandar porque no es solo eso, es toda la retafila que te dice. No hay derecho a que yo vaya a tu consulta y me digas esas barbaridades (T4).

(4) Pero que lleguemos a hospitales y te digan eso: 'Vete a un taller de biodanza. Vete a hacer manualidades'. No, perdona. Yo en mi tiempo libre yo hacía lo que me daba la gana [...]. Pido que me cures y para curarme tendrás que investigar (T4).

(5) Siempre, siempre había una excusa [...]. 'Pues es debilidad' [...] 'Pues es final de año; es una época mala' (T2).

(6) Que te lo digan los amigos ['Qué buena cara tienes'] está bien, pero que te lo digan los médicos como juicio clínico... (T5). 
La afirmación o incluso la insinuación de que los problemas que los pacientes sienten como físicos son vistos más bien como psíquicos remiten igualmente hacia una supuesta infravaloración de la enfermedad y de los enfermos por parte de los profesionales. Los adverbios 'siempre' o 'directamente', la estructura 'llegar a preguntar', la reacción airada ante determinadas preguntas o recomendaciones y el recuerdo de expresiones groseras intensifican el proceso:

(7) Pues siempre se creen que somos anoréxicas. Pero hasta a los hombres les dicen que son anoréxicos y te dicen que es psicológico, cosa que no es así, pero es una pelea continua (T5).

(8) A mí me llegan a preguntar si yo había tenido algún traumatismo emocional (T2).

(9) La última visita al internista porque fui con dolor en todo el cuerpo me dice 'Has tenido algún ( $\mathrm{xxx}$ ) traumática o algún disgusto estos tres meses?'. Y yo digo 'Sí, ¿y qué le importa? [...]. 'Sí, ¿y a usted qué le importa?'. No, es que lo comenté así porque me cabreó con todo el dolor del mundo, que no hay manera de que se vaya el dolor de nada, que no puedo andar y me pregunta si he tenido trauma familiar. Digo 'Y a usted le importa algo?' (T7).

(11) Yo, en $\mathrm{La} \mathrm{Fe}$, intenté que me vieran y directamente me querían meter en la planta de psiquiatría de anoréxicas. Y estaba diagnosticada desde hacía 7 años (T5).

(10) Una persona con sensibilidad química múltiple, que es amiga mía, le dijeron 'A ti lo que te hace falta es un buen polvo'. A mí me dijeron que yo tenía que subir en bicicleta para sobrevivir a mi dolor (T5).

La infravaloración conduce a errores diagnósticos, como también ocurre con la sobrevaloración (Moynihan et al., 2012; Moynihan, 2012). Curiosamente, en ambos casos podemos desembocar en un exceso de diagnósticos o en un exceso de medicamentos tomados:

(12) Pasaron 4, 5 o 6 años por lo menos, que primero me dijeron esclerosis múltiple porque en la punción lumbar salía esclerosis múltiple. Me trataron para la esclerosis y al cabo de 2 o 3 años decidieron otra punción lumbar y salió que no era esclerosis múltiple [...]. Y una pregunta por casualidad: ¿Tú tienes llagas en la boca? (T7).

(13) Yo he pasado por tener mononucleosis, por tener afectación del nervio que pasa por aquí, que ya ni me acuerdo, quiero decir, me han hecho pruebas de tuberculosis, SIDA... (T2). 
(14) Yo, en mi caso, ahora tengo uno y medio porque dicen que también tengo el tema de la fibromialgia, aunque yo no lo-, conozco a gente con fibromialgia y no me siento muy, muy identificada con ellas, pero diagnosticada estoy de las dos (T.4).

(15) Te inflan a medicación muy fuerte toda ella que no te resuelve absolutamente nada (T2).

La utilización de marcos metafóricos relacionados con la batalla frente a la enfermedad es algo frecuente en enfermedades graves, como lo son la mayor parte de las poco frecuentes. Pero cuando esos marcos están dirigidos hacia la relación asimétrica entre médico y paciente, hacia la gestión del alta, el acceso a medicamentos o la falta de modestia, la interpretación es bien distinta como puede apreciarse en estas afirmaciones de una de nuestras informantes:

(16) O sea, es David contra Goliat. Pero hay que luchar y cuando uno te dice 'Te doy el alta', yo voy a por el siguiente. No hay que rendirse (T5).

(17) Buscan que te rindas. Consciente algunos, inconscientemente otros. Normalmente es el propio prurito profesional. No sé más y como no sé más te digo que no hay más, ¿no? No di-, no digas 'No hay más', di 'No sé más’ (T5).

(18) Entonces, hasta para lo que funciona me tengo que pelear (T5).

Pero en las enfermedades poco frecuentes, en las que el perfil de paciente experto no es algo anecdótico ni mucho menos, la demostración del modelo asimétrico se aprecia con fuerza cuando alguno de los testigos recuerda la falta de disposición de los profesionales sanitarios a aceptar una conversación pausada en torno a novedades terapéuticas:

(19) Cuando tú vas y le dices 'Oye, esta investigación, esta sentencia, esto, esto, esto', él se siente agredido. Te dice que tú quién eres para ir allí a contarle eso (T4).

En estas enfermedades hablamos también de pacientes pluripatológicos, que han de acudir a numerosas consultas médicas. Buscan una interacción reticular en la que esté presente una prueba de que esos distintos especialistas implicados están coordinados entre sí y que haya homogeneidad en sus mensajes (Patsos, 2001: 805; Bensing et al., 2003: 30-31); cosa que no siempre sucede. 'Ir por su lado' o 'cada uno por su cuenta' expresan bien el nivel de descoordinación sentido, como también lo hace la estructura 'empezar desde cero' o la palabra 'aberración' al comentar la asunción de responsabilidades de coordinación por parte de la propia paciente. Igual- 
mente, merece una mención especial la repetición de estructuras hipotéticas ( $s i$ ) y de obligación (tener que), el uso de la ironía y los cuantificadores de carácter hiperbólico:

(20) Llegué con mi tocho de historial, lo puse encima de la mesa y la doctora dijo 'Esto a mí me da igual. He de empezar desde cero'. Yo la miré y la dije 'Hasta la próxima, my, my dear', ¿eh? (T5).

(21) Pero, sin criticar al sistema de salud, pero lo han-, no hubo una coordinación entre el, el neumólogo y el digestivo, sino que cada quien va, va por su lado (T6).

(22) Yo tengo que actuar de coordinador de todos mis médicos; yo actúo de coordinadora; eso es una aberración [...] En el extremo de que si voy al hematólogo le tengo que decir lo que ha dicho el digestólogo. Y si voy al ginecólogo, le tengo que decir lo que ha dicho el hematólogo. Y si voy al inmunólogo le tengo que decir lo que ha dicho el neurólogo. Y si, y si, y si... Y a mí me llevan más de una decena de especialidades (T5).

(23) Que esa es otra. Como todos vosotros, del psicólogo al psiquiatra, medicina interna, el, el -¿cómo se dice?- el reumatólogo, bueno tropecientos mil especialistas que cada uno iba por su cuenta. Aún sigue yendo cada uno por su cuenta (T4).

La coordinación entre especialistas y secciones, además, evitaría los casos de autodiagnóstico que, en ocasiones, puede ser una buena manera de gestionar la relación entre médico y paciente (Frankel, 2001), pero que en otras, la mayoría, es vista como prueba de incompetencia profesional:

(24) Yo acabé diagnosticándome a mí misma después de esa gran travesía en el desierto (T5).

(25) Yo también me autodiagnostiqué, como dices tú, porque a mí me estuvieron tratando para lupus. Yo era una luposa superclara. Durante dos años medicación superdura que yo decía ya, como decías tú, 'yo me encuentro peor y peor y peor, y el riñón jodido porque te dan la medicación'. Y luego decía, 'Con lo fácil que ha sido', porque yo luego me metí en Internet -esas cosas que te dicen que no hagas- dije 'Síntoma pla, pla, pla'. Y dije 'Huy, esto coincide con esto'. O sea, así de sencillo (T4).

O el hecho de tener que estar contando siempre la misma historia, algo que para los pacientes es agotador:

(26) En un segundo ingreso tengo la suerte de que me toca en agosto; no había ningún especialista; estaban todos de vacaciones y yo decido que a mí no me toca ya nadie más. Es decir, yo llegué un momento a can- 
sarme de que me hicieran biopsias, de que me hubieran, me biopsiaran absolutamente todo, y decidí que a mí no me va a tocar nadie. Yo ya le había contado toda mi historia a unos médicos y yo no iba a ir repitiéndola continuamente (T2).

(27) O sea, lo que estabas comentando antes del tema de ir a la inspección médica, de contar, de ir al médico, contar, a tal, a cual. O sea, yo ya decía 'Mira, me voy a grabar con un casete y se lo voy a poner', porque es que ya es remover $-\mathrm{y}$ hablando mal- la mierda (T3).

El modelo asimétrico, biomédico, no contempla apenas el intercambio de ideas con el paciente en relación a las distintas pruebas que son posibles para la realización, por ejemplo, de un diagnóstico. Y ese paciente puede encontrar esas alternativas fácilmente al buscar en la Red:

(28) Yo quería, yo quería comentar sobre lo de la antitripsina, que, que lo, los médicos la..., o sea, el digestivo me mandó hacer una biopsia, y yo busco por Internet y dice que sí se detecta un déficit de alfa antitripsina por genética, y él me mandó hacer una biopsia (T6).

También se echa de menos la conversación con respecto al momento sobre el que hay que adoptar medidas teniendo en cuenta que hablamos, en muchos casos, de enfermedades graves y que avanzan, por lo que algunas intervenciones tempranas, preventivas, podrían ser una buena opción. T6 se vale de la interrogación retórica y T5 aprovecha el valor intensificador de la palabra 'fatal' o la estructura de asunción de responsabilidad 'tomar cartas en el asunto':

(29) Pero, ¿cuándo me la ponen? ¿Cuándo me la van a poner? Cuando tenga los pulmones a una capacidad de que yo ya no pueda ni respirar. ¿Por qué no me la pones ahora y mantienes el nivel y mis pulmones están sanos? (T6).

(30) Esperan a que estés fatal para tomar cartas en el asunto (T5).

Si se plantea en términos de súplica, la interacción entre médico y paciente se hace muy evidente:

(31) Tardamos 4 años en dar con un tratamiento para el dolor que me funcionara. Yo tengo que suplicar que me den el centanilo porque sólo se lo dan a los pacientes oncológicos. Y tengo que suplicar que me hagan intervenciones periódicas para que-, para quemarme los nervios esplácnicos (T5).

Es normal hallar expresiones que reflejan bien la repetición de interacciones con distintos médicos, sin que esas interacciones supongan ne- 
cesariamente un avance en el conocimiento de lo que sucede. En otro trabajo identificábamos marcos metafóricos expresados por afectados y familiares entrevistados; tales como peregrinaje, círculo vicioso, laberinto, pozo o túnel (Requena, Arcos y Bañón, 2014). En el discurso de los afectados que participaron en el grupo de discusión nuevas propuestas pudimos observar (pulular, tránsito por el desierto, vagar, puzle, fregadero, cuneta, Himalaya, inmensidad, camino de fondo):

(32) Mi diagnós-, mi diagnóstico ha sido un... pulular por especialistas y por analíticas y por pruebas, por psicólogos, psiquiatras (T7).

(33) Claro, ese período de, de, yo lo veo como el tránsito por el desierto, ¿no? O sea, es ir vagando, vagando de un sitio a otro, de otro a uno (T2).

(34) Es como un puzle, ¿no? Quito, pongo, pongo, cojo, tal. Es un poco así, ¿no? (T2).

(35) Yo viví una gran travesía en el desierto, brutal travesía en el desierto en búsqueda de un diagnóstico. Mientras tanto, toda mi vida se fue por el fregadero (T5).

(36) Si no tienes ciertas capacidades estás, vamos, en la cuneta, en la cuneta absolutamente. Sobre todo, cuanto más sistémica más en la cuneta estás (T5).

(37) Es el Himalaya (T4).

(38) En ningún momento son conscientes eh: de la inmensidad a la que te enfrentas (T5).

(39) Y: sigo teniendo muchos problemas, pero esto es un camino, es de fondo, es de fondo (T5).

En cualquier caso, la muestra más clara de las consecuencias negativas que puede tener un modelo comunicativo únicamente biomédico es la presencia de la mentira en la relación entre médico y paciente. Es el ejemplo más vivo del fracaso comunicativo:

(40) Pero lo triste es que tengo que ir mintiendo a todos los médicos, porque desde el primer día que dije que estaba haciendo un tratamiento experimental, que va con un comité médico, quiero decirte, que no es que nosotros digamos 'Vamos a probar' $[\ldots]$ me dijeron que "no... no te lo vamos a cubrir y si haces ese tratamiento aquí no vengas" [...]. Tuve que mentir a todos y decir a todos que me lo había dejado para poder seguir yendo a consultas y seguir haciéndome pruebas (T4). 


\section{A MODO DE CONCLUSIÓN}

Marisa Cordella (2002) alude a la utilización de los médicos de tres tipos de voces cuando interactúan con pacientes en consultas médicas:

- la voz médica (que busca obtener información de la salud del paciente),

- la voz educativa (que asesora al paciente sobre la condición médica), y

- la voz empática (que presta atención a la identidad social del individuo que carece de buena salud).

Gestionar bien esas voces permite acomodarse al contexto comunicativo y al perfil de los interlocutores (Douglas et al., 2015). En la actualidad, la tendencia a la asunción de mayores responsabilidades por parte de los enfermos y de sus familiares ha de corresponderse, por un lado, con una mayor preparación de éstos, de manera que haya también tres tipos de voces de los pacientes (informativo, mostrativo y colaborativo), y, por otro, de la asunción de responsabilidades por parte de los otros actores esenciales (los profesionales y los gestores públicos, por ejemplo). En cualquier caso, parece que el reto pendiente sigue siendo la incorporación del componente reticular en las entrevistas entre profesionales sanitarios y afectados. Esa incorporación supondría que hubiera una coordinación entre áreas, secciones y profesionales y que la información estuviera actualizada, evitando contradicciones y dispensando a los pacientes y sus familiares de tener que estar repitiendo continuamente sus problemas de salud; repetición que genera, por lo normal, una gran ansiedad.

Las enfermedades poco frecuentes constituyen un área relevante a la hora de observar lo que los pacientes recuerdan sobre el tipo de interacción que desarrollan con sus médicos. Aunque también hay alusiones en el discurso de los testigos analizados en torno a la presencia de patrones de comportamiento próximos al modelo psicosocial o biopsicosocial, hemos podido detectar numerosas alusiones a técnicas de distanciamiento por parte de los profesionales que remiten al modelo más asimétrico. Hay, igualmente, distintas estrategias y herramientas discursivas utilizadas por los pacientes adultos estudiados para intensificar la infravaloración de sus enfermedades, el desconocimiento de las mismas, la falta de asunción de responsabilidades o la ausencia de técnicas de incorporación de los afectados en el proceso comunicativo relacionado con el tratamiento o con el cuidado crónico, lo que hace que sean los propios pacientes los que asuman responsabilidades incluso en el diagnóstico gracias, entre otras cosas, al mayor y mejor acceso a información relevante a través de Internet (Price et al., 2010). 


\section{BIBLIOGRAFÍA}

ÁGREDA, J. y YANGUAS, E. (2001): "El paciente difícil: quién es y cómo manejarlo", Anales del Sistema Sanitario de Navarra, 24 (2), págs. 65-72.

Aibar, C. (2009): “¿Nos creemos de verdad la necesidad de la participación del paciente?”, Revista de Calidad Asistencial, 24 (5), págs. 183-184.

Ainsworth-Vaughn, N. (2003): "The discourse of medical encounters", en D. Schiffrin, D. Tannen y H. Hamilton (eds.), The Handbook of Discourse Analysis, Malden, Blackwell, págs. 453-469.

Amer, A. y Fischer, H. (2009): "Don't call me 'mom'”: How parents want to be greeted by their pediatrician”, Clinical Pediatrics, 48 (7), págs. 720-722.

Aper, L., Veldhuijzen, W., Dornan, T., van de Ridder, M., Koole, S., Deresea, A. y RENIERS, J. (2015): “'Should I prioritize medical problem solving or attentive listening?': The dilemmas and challenges that medical students experience when learning to conduct consultations", Patient Education and Counseling, 98 (1), págs. 77-84.

BAÑón, A. M. (2007): "Las enfermedades raras y su representación discursiva. Propuestas para un análisis crítico”, Discurso \& Sociedad, 1 (2), págs. 188-229.

BAÑón, A. M. y ForNieles, J. (2011): "Comunicación y Enfermedades Raras: contextualización”, en A. M. Bañón et al. (eds.), Desafíos y estrategias comunicativas de las enfermedades raras: la investigación médica como referente. Valencia, CIBERER, págs. 11-40.

BEACH, W. A. (1995): "Preserving and constraining options: 'okays' and 'official' priorities in medical interviews", en G. H. Morris y R. Chenail (eds.), The Talk of the Clinic: Explorations in the Analysis of Medical and Therapeutic Discourse, Lawrence Erlbaum, Hillsdale, New Jersey, págs. 259-289.

Beckman, H. y Franjel, R. (1984): "The effect of physician behavior on collection of date”, Annals of Internal Medicine, 101 (5), págs. 692-696.

Bellón, J. A. (2001): "Habilidades de comunicación y utilización de las consultas de atención primaria”, Anales del Sistema Sanitario de Navarra, 24 (2), págs. $7-14$.

BennetT, K. e IRwin, H. (1997): "Shifting the emphasis to 'patient as central': Sea change or ripple on the pound?”, Health Communication, 9 (1), págs. 83-93.

Bensing, J., van Dulmen, S. y Tates, K. (2003): "Communication in context: new directions in communication research", Patient Education and Counseling, 50, págs. 27-32.

BerEsford, B. y SLOPER, P. (2003): “Chronically ill adolescents' experiences of communicating with doctors: a qualitative study”, Journal of Adolescent Health, 33, págs. 172-179.

BÉRY, A. (2005): "Information et consentement", EMC-Odontologie, 1, págs. 262280. 
Blanck, P., Rosenthal, R. y Vannicelli, M. (1986): "Talking to and about patients: the therapist's tone of voice”, en D. Blanck, R. Buck y R. Rosenthal (eds.), Nonverbal Communication in the Clinical Context, The Pennsylvania State University Press, págs. 99-143.

Borrell, F. (2004a): Entrevista clínica. Manual de estrategias prácticas, SemFYC, Barcelona.

Borrell, F. (2004b): "Entrevista clínica viva", Atención Primaria, 34 (1), págs. 29-31.

BORRELL, F. y EPSTEIN, R. M. (2004): "Preventing errors in clinical practice: a call for self-awareness", Anuals of Family Medicine, 2 (4), págs. 310-316.

Borrell, F., Hernández, J. C. y LÁzaro, J. (2011): "Demanda y queja en la entrevista clínica, conceptos clave para una práctica clínica segura”, Medicina Clínica, 137 (5), págs. 216-220.

Britten, N., Stevenson, F., Gafaranga, J., Barry, Ch. y Bradley, C. (2004): "The expression of aversion to medicines in general practice consultations", Social Science \& Medicine, 59, págs. 1495-1503.

Budych, K., Helms, Th. y Schultz, C. (2012): "How do patients with rare diseases experience the medical encounter? Exploring role behavior and its impact on patient-physician interaction", Health Policy, 105, págs. 154-164.

Candlin, C. (2000): "Reinventing the patient/client: new challenges to healthcare communication”, The Cardiff Lecture. Accesible en: <www.cf.ac.uk/encap/ HCRC/candlin.lecture.pdf $>$.

CASSELl, E. J. (1989): "Making the subjective objective”, en M. Stewart y D. Roter (eds.), Communicating with medical patients, London, Sage, págs. 13-15.

Cegala, D. J., Post, D. y McClure, L. (2001): "The effects of patient communication skills training of the discourse of older patients during a primary care interview", Journal of the American Geriatrics Society, 49 (11), págs. 1505-1511.

Charles, C., Gafni, A. y Whelan, T. (1999): "Decision-making in the physicianpatient encounter: revisiting the shared treatment decision-making model", Social Science \& Medicine, 49, págs. 651-661.

Chenail, R. y Morris, G. H. (1995): "Introduction: the talk of the clinic", en G. H. Morris y R. Chenail (eds.), The talk of the clinic. Explorations in the analysis of medical and therapeutic discourse, Hillsdale, Lawrence Erlbaum Associates, págs. 1-15.

Chesser, A., Keene, N., Davis, A. y Bowers, C. (2012): "Prescribing information therapy: opportunity for improved physician-patient communication", Journal of Primary Care \& Community Health, 3 (1), págs. 6-10.

Chiong, W. (2004): "Diagnosing and defining disease", en A. Caplan, J. McCartney y D. Sisti (eds.), Health, disease and illness. Concepts in medicine, Washington, Georgetown University Press, págs. 128-131.

Clark, J. A. y Mishler, E. G. (1992): “Attending to patients' stories: reframing the clinical task”, Sociology of Health \& Illness, 14 (3), págs. 344-372.

ConTe, R. (1981): "Aspects of interaction in a medical interview", Journal of Pragmatics, 5, págs. 113-144.

Cordella, M. (2000): "Medical discourse in a Hispanic environment: Power and simpatia under examination", Australian Review of Applied Linguistics, 22 (2), págs. 35-50. 
CoRdella, M. (2002): "La interacción médico-paciente en escrutinio: un estudio de sociolingüística interaccional”, Onomazein, 7, págs. 117-144.

Cornellá, J. y Llusent, A. (2002): "La relación médico-paciente", Anales del Sistema Sanitario de Navarra, 25 (2), págs. 145-149.

Coupland, N., Coupland, J., Giles, H. y Henwood, K. (1988): “Accommodating the elderly: Invoking and extending a theory”, Language in Society, 17, págs. $1-41$.

Coupland, J., Robinson, J. D. y Coupland, N. (1994): "Frame negotiation in doctor elderly patient consultation”, Discourse \& Society, 5 (1), págs. 89-124.

Cushing, A. (2016): "History of the doctor-patient relationship", en J. Brown, J. Kidd, N. Lorraine y A. Papageorgiou (eds.), Clinical Communication in Medicine, John Willey \& Sons, Oxford, págs. 6-20.

Di Ruggiero, E., Cohen, J., Cole, D. y Forman, L. (2015): “Competing conceptualizations of decent work at the intersection of health, social and economic discourses”, Social Science \& Medicine, 133, págs. 120-127.

Dobson, R. (2002): "Sharing of uncertainty can unnerve patients", British Medical Journal, 325, pág. 1319.

Douglas, C., Wilcox, E. Burgess, M. y Lynd, L. (2015): "Why orphan drug coverage reimbursement decision-making needs patient and public involvement”, Health Policy, 119, págs. 588-596.

Eliott, J. y Olver, I. (2002): “The discursive properties of 'hope': A qualitative analysis of cancer patients' speech”, Qualitative Health Research, 12 (2), págs. 173-193.

Elwyn, G., Gwyn, R., Edwards, A. y Grol, R. (1999): "Is 'shared decision-making' feasible in consultations for upper respiratory tract infections? Assessing the influence of antibiotic expectations using discourse analysis", Health Expectations, 2, págs. 105-117.

ENGEL, G. L. (1977): "The need for a new medical model: A challenge for biomedicine”, Science, 196 (4286), págs. 129-136.

Engeström, Y., Engeström, R. y Kerosuo, H. (2003): "The discursive construction of collaborative care”, Applied Linguistics, 24 (3), págs. 286-315.

FALlowfield, L. y Jenkins, V. (2004): "Communicating sad, bad, and difficult news in medicine”, The Lancet, 363, págs. 312-319.

FEDER (2009): Estudio de necesidades sociosanitarias de las personas con enfermedades raras en España (ENSERIO), Madrid, Cajamadrid.

FEDER (2017): Estudio de necesidades sociosanitarias de las personas con enfermedades raras en España (ENSERIO III), Valencia, Observatorio de las Enfermedades Raras de FEDER. En prensa.

FIRTH, M. (1983): "Diagnosis of Duchenne Muscular Dystrophy: Experiences of parents of sufferers", British Medical Journal, 286 (6366), págs. 700-701.

Fisher, S. y Groce, S. (1985): "Doctor-patient negotiation of cultural assumptions", Sociology of Health \& Illness, 7 (3), págs. 342-374.

Frankel, R. (1990): "Talking in interviews: a dispreference for patient iniciated questions in physician-patient encounters", en G. Psathas (ed.), Interaction competence, Lanham, M. D., University Press of America, págs. 231-261.

FrAnkEL, R. (2001): "Clinical care and conversational contingencies: The role of patients' self-diagnosis in medical encounters”, Text, 21 (1/2), págs. 83-111. 
Frankel, R. y BeckMAN, H. (1989): "Evaluating the patient's primary problem(s)", en M. Stewart y D. Roter (eds.), Communicating with medical patients, London, Sage, págs. 86-98.

GADAMER, H. G. (2001): El estado oculto de la salud, Barcelona, Gedisa.

Gómez, M. (2000): Cómo dar las malas noticias en medicina, Madrid, Arán Ediciones.

GwYN, R. (2001): Communicating Health and Illness, London, Sage.

HAAKANA, M. (2001): "Laughter as a patient's resource: dealing with delicate aspects of medical interaction”, Text, 21 (1/2), págs. 187-219.

HAAKANA, M. (2002): "Laughter in medical interaction: from quantification to analysis, and back", Journal of Sociolinguistics, 6 (2), págs. 207-235.

Hall, D. E., Prochazka, A. V. y Fink, A. S. (2012): "Informed consent for clinical treatment”, Canadian Medical Association Journal, 184 (5), págs. 533-540.

Halpin, M. (2011): "Diagnosis, psychiatry and neurology: The case of Huntington Disease”, Social Science \& Medicine, 73, págs. 858-865.

Heath, C. (1986): Body movement and speech in medical interaction, Cambridge, Cambridge University Press.

HeAth, C. (1989): "Pain talk: The expression of suffering in the medical consultation”, Social Psychology Quarterly, 52, págs. 113-125.

HenzL, V. M. (1990): "Linguistic means of social distancing in physician-patient communication", en W. von Raffler-Engel (ed.), Doctor-Patient Interaction, Amsterdam, John Benjamins Publishing Company, págs. 77-91.

Hsieh, E., Bruscella, J., Zanin, A. y Kramer, A. (2016): “'It's not like you need to live 10 or 20 years': Challenges to patient-centered care in gynecologic oncologist-patient interactions", Qualitative Health Research, 26 (9), págs. 11911202.

Hydén, L. y Mishler, E. (1999): "Language and Medicine", Annual Review of Applied Linguistics, 19, págs. 174-192.

JonEs, CH. (2001): "Missing assessments: Lay and professional orientations in medical interviews”, Text, 21 (1/2), págs. 113-150.

Koch-Weser, S., Dejong, W. y Rudd, R. E. (2009): "Medical word use in clinical encounters”, Health Expectations, 12, págs. 371-382.

Korsch, B. M. (1989): "The past and the future of research in doctor-patient relations", en M. Stewart y D. Roter (eds.), Communicating with medical patients, London, Sage, págs. 246-250.

Krosin, M. T., Klitzman, R., Levin, B., Cheng, J. y Ranney, M. L. (2006): "Problems in comprehension of informed consent in rural and peri-urban Mali, West Africa”, Clinical Trials, 3, págs. 306-313.

Kushner, T. (1981): "Doctor-patient relationships in general practice- a different model”, Journal of Medical Ethics, 7, págs. 128-131.

LABov, W. y FAnshel, D. (1977): Therapeutic discourse: Psychotherapy as conversation, New York, Academic Press.

LAín, P. (1964): La relación médico/enfermo. Historia y teoría, Revista de Occidente, Madrid.

LAín, P. (1982): El diagnóstico médico. Historia y teoría, Salvat, Barcelona.

LEE, H. (2002): "Longer consultations can improve patient satisfaction", British Medical Journal, 325, pág. 1241. 
Levenstein, J. H., Brown, J. B., Weston, W., Stewart, M., McCracken, E. C. y McWhinney, I. (1989): "Patient-centered clinical interviewing", en M. Stewart y D. Roter (eds.), Communicating with medical patients, London, Sage, págs. 107120.

Lizarraga, S. y Ayarra, M. (2001): "Entrevista motivacional", Anales del Sistema Sanitario de Navarra, 24 (2), págs. 43-53.

Maguire, P. y Pitceathly, C. (2002): "Key communication skills and how to acquire them", British Medical Journal, 325, págs. 697-700.

Majerovitz, S., Green, M., Adelman, R., Brody, G., Leber, K. y Healy, S. (1997): "Older Patients' understanding of medical information in the Emergency Department”, Health Communication, 9 (3), págs. 237-251.

Malson, H., Finn, D. M., Treasure, J., Clarke, S. y Anderson, G. (2004): "Constructing 'The eating Disordered Patient': a discourse analysis of accounts of treatment experiences", Journal of Community \& Applied Social Psychology, 14, págs. $473-489$.

MaynaRd, D. y Heritage, J. (2005): "Conversation analysis, doctor-patient interaction and medical communication”, Medical Education, 39, págs. 428-435.

MCWhinNeY, I. R. (1989): "The need for a transformed clinical method", en M. Stewart y D. Roter (eds.), Communicating with medical patients, London, Sage, págs. 25-57.

MikeSell, L. y Bromley, E. (2012): "Patient centered, nurse averse? Nurses' care experiences in a 21st- century hospital”, Qualitative Health Research, 22 (12), págs. 1659-1671.

Morant, R. (2016): "La antroponimia en la atención sanitaria”, Círculo de Lingüistica Aplicada a la Comunicación, 66, págs. 278-300.

Mordacci, R. y Sobel, R. (2004): "Health: A comprehensive concept", en A. Caplan, J. McCartney y D. Sisti (eds.), Health, disease and illness. Concepts in medicine, Washington, Georgetown University Press, págs. 104-109.

Moynihan, R. (2012): "When less can mean more", The Medical Journal of Australia 196 (9), pág. 555.

Moynihan, R., Doust, J. y Henry, D. (2012): "Preventing overdiagnosis: how to stop harming the healthy", British Medical Journal, 344, pág. 3502.

Murtagh, G. M., Furber, L. y Thomas, A. L. (2013): "Patient-initiated questions: How can doctors encourage them and improve the consultations process? A qualitative study", BMJ Open, 3 (10), págs. 1-6.

Nessa, J. y Malterud, K. (1998): “Tell me what's wrong with me: A discourse analysis approach to the concept of patient autonomy", Journal of Medical Ethics, 24, págs. 394-400.

Noble, L. (2016): "The future of the doctor-patient relationship", en J. Brown, J. Kidd, N. Lorraine y A. Papageorgiou (eds.), Clinical Communication in Medicine, John Willey \& Sons, Oxford, págs. 57-64.

Olson, K., ZimkA, O. y STEIn, E. (2015): "The nature of fatigue in chronic fatigue syndrome”, Qualitative Health Research, 25 (10), págs. 1410-1422.

Opsal, T., Wolgemuth, J., Cross, J., KaAnta, T., Dickmann, E., Colomer, S. y ERdiL-Moody, Z. (2016): “'There are no know benefits...': Considering the risk/benefit ratio of qualitative research", Qualitative Health Research, 26 (8), págs. 1137-1150. 
Papageorgiou, A. (2016): "Models of the doctor-patient consultation", en J. Brown, J. Kidd, N. Lorraine y A. Papageorgiou (eds.), Clinical Communication in Medicine, John Willey \& Sons, Oxford, págs. 21-29.

Patsos, M. (2001): "The Internet and Medicine: Building a community for patients with Rare Diseases", JAMA, 285 (6), pág. 805.

Peters, S., Stanley, I., Rose, M. y Salmon, P. (1998): "Patients with medically unexplained symptoms: Sources of patients' authority and implications from demands on medical care”, Social Science \& Medicine, 46 (4/5), págs. 559-565.

Price, J., Shen, L., Robinson, J. D. y Farrell, Ph. (2010): "Parental information seeking following a positive newborn screening for Cystic Fibrosis", Journal of Health Communication, 15 (8), págs. 888-894.

Prieto, J. F. (2001): "Palabra, palabrería y verdad en el discurso médico", Logos. Revista de Retórica y Teoría de la Comunicación, 1 (2), págs. 1-2.

Requena, S., Arcos, J. M. y Bañón, A. M. (2014): "Cómo recuerdan los pacientes la comunicación del diagnóstico. A propósito de quince testimonios relacionados con enfermedades poco frecuentes", Cultura, Lenguaje y Representación, 13, págs. 165-184.

Requena, S. y BAÑón, A. M. (2016): "El discurso solidario. Mensajes enviados a la Telemaratón de TV3 a favor de las personas con enfermedades poco frecuentes”, CLAC. Círculo de Lingüística Aplicada a la Comunicación, 65, págs. 317-355.

Rivera-Navarro, J. (2009): “The diagnosis of Tourette's Syndrome: Communication and impact”, Clinical Child Psychology and Psychiatry, 14 (1), págs. 13-23.

Robinson, J. D. (2001): "Asymmetry in action: Sequential resources in the negotiation of a prescription request", Text, 21, págs. 19-54.

Roscigno, C., Savage, T., Kavanagh, K., Moro, R., Kilpatrick, S., Strassner, H., Grobman, W. y Kimura, R. (2012): "Divergent views of hope influencing communications between parents and hospital providers", Qualitative Health Research, 22 (9), págs. 1232-1246.

Schaepe, K. (2011): "Bad news and first impressions: Patient and family caregiver accounts of learning the cancer diagnosis”, Social Science \& Medicine, 73, págs. 912-921.

Schmidt, H. G., Van Gogh, T., Schuit, S., Van den Berge, K., Van Daele, P., Bueving, H., Van der Zee, T., Van den Broek, W., Van Saase, J. y Mamede, S. (2017): "Do patients' disruptive behaviours influence the accuracy of a doctor's diagnosis? A randomised experiment”, BMJ Quality and Safety, 26, págs. 19-23.

Servicio de Epidemiología de la Comunidad de Madrid (2008): "Mortalidad y años potenciales de vida perdidos por enfermedades raras en la Comunidad de Madrid en el periodo 1999-2003”, Boletín Epidemiológico de la Comunidad de Madrid, 2 (14), págs. 3-41.

Sharf, B. y Street, R. (1997): “The patient as a central construct: Shifting the emphasis", Health Communication, 9 (1), págs. 1-11.

Silverman, D. (1987): Communication and Medical Practice, London, Sage.

Starke, M., Albertsson-Wikland, K. y Möller, A. (2002): "Parents' experiences of receiving the diagnosis of Turner Syndrome: an explorative and retrospective study", Patient Education and Counseling, 47, págs. 347-354. 
Stivers, T. y Heritage, J. (2001): "Breaking the sequential world: Answering 'more than the question' during comprehensive history taking", Text, 21 (1/2), págs. 151-185.

STRATFORD, J. (1998): "Women and men in conversation: A consideration of therapists' interruptions in therapeutic discourse", Journal of Family Therapy, 20, págs. 383-394.

Street, R. (1991): “Accommodation in medical consultations", en H. Giles, J. Coupland y N. Coupland (eds.), Contexts of Accommodation. Developments in Applied Sociolinguistics, Cambridge, Cambridge University Press, págs. 131-156.

Swenson, S., Buell, S., Zettler, P., White, M., Ruston, D. y Lo, B. (2004): "Patientcentered communication. Do patients really prefer It?", Journal of General Internal Medicine, 19, págs. 1069-1079.

Ten Have, P. (1991): “Talk and institution: A reconsideration of the 'Asymmetry' of Doctor-Patient Interaction”, en D. Boden y D. H. Zimmerman (eds.), Talk and Social Structure. Studies in Ethnomethodology and Conversation Analysis, Polity Press, Oxford, págs.138-163.

Ten Have, P. (1995): "Medical ethnometodology: An overview", Human Studies, 18, págs. 245-261.

Thompson. T. L. (1990): "Patient health care: Issues in Interpersonal Communication”, en E. Berlin y L. Donohew (eds.), Communication and Health. Systems and Applications, Hillsdale, Lawrence Erlbaum Associates, págs. 27-50.

Thompson, C. y Pledger, L. (1993): "Doctor-patient communication: Is patient knowledge of medical terminology improving?", Health Communication, 5 (2), págs. 89-97.

Treichler, P., Frankel, R. M., Kramarae, C., Zoppi, K. y Beckman, H. B. (1984): "Problems and problems. Power relationships in a medical encounter", en C. Kramarae, M. Schulz y W. M. O’Barr (eds.), Language and Power, Beverly Hills, Sage, págs. 62-83.

Van Dulmen, A. M. (2002): "Different perspectives of doctor and patient in communication”, International Congress Series, 1241, págs. 243-248.

VON RAFFLER-Engel, W. (1989): "Doctor-patient relationships in the 1980", en W. von Raffler-Engel (ed.), Doctor-patient interaction, Amsterdam, John Benjamins Publishing, págs. 1-43.

Weston, W. y LIPKIN, M. (1989): "Doctors learning communication skills: developmental issues", en M. Stewart y D. Roter (eds.), Communicating with medical patients, London, Sage, págs. 43-57.

ZopPI, K. y EPsteIn, R. M. (2002): "Is Communication a skill? Communication behaviors and being in relation", Communication Techniques and Behaviors, 34 (5), págs. 319-324. 